Proceedings

\title{
Assessment of Occupational Stress Index and Lipid Profile among Professional Drivers in Ismailia City, Egypt $^{\dagger}$
}

\author{
Sarah M. Hussein 1,*, Rasha F. Abdellah ${ }^{1}$, Essam M. Abdalla ${ }^{2}$, Amani Waheed ${ }^{1}$ \\ and Adel M. Mishriky ${ }^{1}$ \\ 1 Department of Public health, Community Medicine, Environmental Medicine and Occupational Medicine , \\ Faculty of Medicine, Suez Canal University, Ismailia 41522, Egypt; rashaf11@yahoo.com (R.F.A.); \\ amaniwaheed@yahoo.com (A.W.); adel.mishriky@gmail.com (A.M.M.) \\ 2 Department of clinical pathology, Faculty of Medicine, Suez Canal University, Ismailia 41522, Egypt; \\ emabdalla@gmail.com \\ * Correspondence: drsarahhussien@yahoo.com \\ + Presented at the 1st International Electronic Conference on Environmental Health Sciences, \\ 15 November-7 December 2018; Available online: https://iecehs-1.sciforum.net/.
}

Published: 14 November 2018

\begin{abstract}
Background: Driving is a stressful job. Professional drivers are high risk group for occupational stress. Occupational stress has been associated with abnormal levels of lipids. However, many studies could not find any association. Aim and Objectives: The study aimed at contributing to improving the professional drivers' health through assessing occupational stress, lipid profile and their association. Subjects and Methods: It was a cross-sectional study conducted at Suez Canal Authority in Ismailia City, Egypt, including 131 professional drivers. A structured interview questionnaire was used to assess occupational stress index (OSI) and cardiovascular risk factors. Lipid profile in form of cholesterol, triglyceride, low density lipoprotein cholesterol (LDL-C) and high-density lipoprotein cholesterol (HDL-C) were assessed. Results: The total OSI score was $79.98 \pm 6.14$. The prevalence of dyslipidemia was $79.4 \%, 51.9 \%$ of drivers had hypercholesterolemia, $37.4 \%$ had hypertriglyceridemia, $50.4 \%$ had high level of LDL-C, and $45 \%$ had low level of HDL-C. The total OSI score and OSI aspects did not have statistically significant relationship with dyslipidemia. The high demand aspect score of OSI had statistically significant relationship with hypercholesterolemia. The conflict aspect had statistically significant relationship with high LDL-C. The noxious exposure and conflict aspects of OSI had weak positive significant correlations with cholesterol level $(\mathrm{r}=0.163,0.162$ respectively). A weak positive significant correlation $(r=0.149)$ was found between noxious exposure aspect score and LDL-C level. Binary logistic regression analysis was conducted to assess independent risk factors of dyslipidemia. The model included aspects of OSI, total OSI score, driving hours a day, smoking status, passive smoking, body mass index (BMI) and dietary habits. It identified conflict aspect of OSI, driving hours a day, and BMI as predictors of dyslipidemia. Conclusion: Professional drivers are exposed to occupational stress, and dyslipidemia is prevalent among them. Total OSI score does not have statistically significant relationship with dyslipidemia. However, certain aspects of OSI have significant relationship with abnormal lipid profile.
\end{abstract}

Keywords: professional drivers; occupational stress index; lipid profile 


\section{Introduction}

Despite the fact that occupational stress is not a new phenomenon, it becomes progressively globalized and has effects on workers, families and overall society. It is in constant growth and presents a growing medical and economical problem [1]. This stress may occur due to the responsibilities related to the work itself, or conditions that are based on personality conflicts or corporate culture. It can affect individuals' wellbeing if not managed appropriately [2]. Driving is a stressful job which needs high levels of attention and caution if drivers are to cope appropriately with job demands. Driver stress can also occur due to timetables and working hours, traffic and weather conditions, passenger and goods safety, and responsibility if an error and/or an accident occur [3]. Cardiovascular diseases (CVDs) of professional drivers remain a key concern in clinical practice and occupational health research [4]. The risk of cardiovascular events has already been well documented among professional drivers [5]. Occupation is a major socioeconomic factor that together with a prolonged exposure to stress at workplace may have a direct effect on the autonomic nervous system and neuroendocrine activity, which may lead to increased incidence of diabetes mellitus, development of hypertension, and lipid disorders [6].

Professional drivers are exposed to several occupational risk factors such as long working hours, shift work, noise, carbon monoxide, and chemical materials. These factors increase the probability of emerging CVDs. Moreover, these drivers are more prone to developing obesity as they burn fewer calories due to the nature of the work activities, eating poor and irregular diets and driving in a sitting position for long durations every day [5]. Dyslipidemia is a well-established modifiable cardiovascular risk factor [7]. It is a heterogeneous disorder which may occur due to multiple etiologies. Most dyslipidemia is secondary to dietary habits and lifestyle [8]. The risk factors of dyslipidemia include age, smoking, genetics, diet, physical activity, and stress [9]. Although cross-sectional studies have linked occupational stress with lipid disorders, this association is still not consistent [4].

\section{Methods}

This cross-sectional study was performed during 2016-2017 in Suez Canal Authority, Ismailia City, on 131 professional drivers (44 car drivers, 43 bus drivers, and 44 truck drivers) selected randomly. The conclusion criterion was spending at least one year as professional driver. A structured interview questionnaire was prepared by the researcher. It included socio-demographic data, occupational history, medical history, Occupational Stress Index (OSI) and cardiovascular risk factors.

\subsection{Occupational Stress Index}

Permission of the first author Karen Belkić was taken before using this questionnaire. The OSI is arranged as a two-dimensional matrix, the vertical axis being composed of level of information transmission and the stressor aspects placed along the horizontal axis. It was developed also from a cognitive ergonomics perspective. Within the OSI the work environment is seen as a whole, including work schedule, task level concerns, broader organizational factors and physical and chemical exposures. The elements are equally weighted, scored from $0-2$ (maximum), from absent to strongly present, with higher scores meaning higher level of burden. The elements are summed to yield aspects of seven groups: high demands, strictness, underload, extrinsic time pressure, noxious exposure, avoidance, and conflict. The total score of each aspect is calculated by adding input, output, central, and general scores of this aspect. The total OSI score is obtained by adding the total of the seven aspects together [10].

\subsection{Cardiovascular Risk Factors Assessment}

Cardiovascular risk factors were assessed to investigate risk factors other than occupational stress that may affect lipid profile, such as lifestyle (including active and passive smoking, sports practice, and environment), sleep, life stress, medical history, family history of CVD, blood sugar, 
and dietary habits. These factors were evaluated and scored according to Sydney cardiovascular risk assessment questionnaire [11].

\subsection{Clinical examination}

Blood pressure was measured for all participants. The participant was considered as having normal blood pressure if the systolic blood pressure was less than $120 \mathrm{mmHg}$ and diastolic less than $80 \mathrm{mmHg}$. Prehypertension or at risk was considered at systolic blood pressure 120-139 mmHg and diastolic $80-89 \mathrm{mmHg}$. High blood pressure was considered at systolic blood pressure $140 \mathrm{mmHg}$ or higher and diastolic $90 \mathrm{mmHg}$ or higher [12].

Body mass index (BMI) was calculated after measuring height and weight. Below 18.5 was considered underweight; $18.5-<25$ normal or healthy weight; $25.0-<30$ overweight; thirty or above obese [13].

\subsection{Determination of Lipid Profile}

After 12 hours of fasting, a venous blood sample $(5 \mathrm{~mL})$ was taken. Quantitative determination of the lipid profile of participants including total cholesterol (TC), triglycerides (TG), high-density lipoprotein (HDL) cholesterol, and low-density lipoprotein (LDL) cholesterol, after centrifugation of the blood separation of serum, was done [14]. Dyslipidemia was operationally defined by one or more of the following abnormalities: Total cholesterol $>200 \mathrm{mg} / \mathrm{dL}$, triglycerides $>150 \mathrm{mg} / \mathrm{dL}$, LDL-C $>130 \mathrm{mg} / \mathrm{dL}$, HDL-C $<40 \mathrm{mg} / \mathrm{dL}$ in men; hypercholesterolemia: Total cholesterol $>200$ $\mathrm{mg} / \mathrm{dL}$; hypertriglyceridemia: triglycerides > $150 \mathrm{mg} / \mathrm{dL}$; hypoHDLaemia: HDL-C < $40 \mathrm{mg} / \mathrm{dL}$; hyperLDLaemia: LDL-C > $130 \mathrm{mg} / \mathrm{dL}$ [15].

\subsection{Statistical Analysis}

All statistical analyses were performed using the Statistical Package for Social Science (SPSS) version 20.0. Data were coded and entered into the computer statistical program and presented as means, standard deviations, and medians for quantitative data. Percentages were computed for categorical data. Tabulation or graphical presentations were done as required. Chi-squared tests were computed for categorical variables. Student $t$-test was used for quantitative variables between two groups and ANOVA test for more than two groups; corresponding non-parametric tests were used for non-parametric data as suitable. Logistic regression analyses for risk factors of dyslipidemia were done. Statistical significance was set at $p<0.05$.

\section{Results and Discussion}

Regarding sociodemographic characteristics of participants, Table 1 shows that the mean age of the studied drivers was $39.8 \pm 8.3$ years, ranging from 27 to 59 years. The age of about half of drivers $(55.7 \%)$ was between $30<40$ years. The highest percentage $(69.5 \%)$ had completed secondary or vocational school. Most of them (93.1\%) were married.

Table 1. Sociodemographic characteristics of the studied drivers $(n=131)$.

\begin{tabular}{lcc}
\hline \multicolumn{1}{c}{ Sociodemographic Characteristics } & No. & $\mathbf{\%}$ \\
\hline Age (years) & \multicolumn{2}{c}{3.8} \\
$20-$ & 53 & 55.7 \\
$30-$ & 31 & 23.7 \\
$40-$ & 22 & 16.8 \\
$50-60$ & \multicolumn{2}{c}{$27-59$} \\
Range & $39.7 \pm 8.3$ \\
Mean \pm SD & \multicolumn{2}{|}{} \\
Educational level & 12 & 9.2 \\
No formal education & \multicolumn{2}{c}{} \\
\hline
\end{tabular}




\begin{tabular}{lcc}
\hline Primary or preparatory & 15 & 11.4 \\
Secondary or vocational school & 91 & 69.5 \\
University & 13 & 9.9 \\
Marital status & & \\
Single & 5 & 3.8 \\
Married & 122 & 93.1 \\
Divorced or widow & 4 & 3.1 \\
\hline
\end{tabular}

Nearly half of the studied drivers (47.3\%) drive both inside the city and between cities. The average duration of driving hours per day was $7.2 \pm 2.6$ hours a day with median of eight hours (Table 2).

Table 2. Occupational history of the studied drivers $(n=131)$.

\begin{tabular}{lcc}
\hline \multicolumn{1}{c}{ Occupational history } & No. & $\%$ \\
\hline City & 36 & 27.5 \\
Inside the city & 33 & 25.2 \\
Between cities & 62 & 47.3 \\
Both & \multicolumn{2}{c}{$1-14$} \\
Average duration of driving hours per day & \multicolumn{2}{c}{$7.2 \pm 2.6$} \\
Range & \multicolumn{2}{c}{8.0} \\
Mean \pm SD & \multicolumn{2}{c}{$1-40$} \\
Median & \multicolumn{2}{c}{$1-40$} \\
Years as professional driver & $14.1 \pm 9.4$ \\
Range & \multicolumn{2}{c}{11.0} \\
Mean \pm SD & \\
Median & \\
\hline
\end{tabular}

Table 3 describes the different aspects of OSI and the total OSI score among the studied drivers. The mean scores of underload, high demand, strictness, extrinsic time pressure, noxious exposure, symbolic aversiveness, and conflict aspects of stress were $(9.56 \pm 2.58,16.92 \pm 2.58,17.22 \pm 1.61,7.56 \pm$ $1.38,6.75 \pm 1.29,9.22 \pm 1.58,12.76 \pm 2.04)$ respectively. The total OSI mean score was $79.98 \pm 6.14$ among them.

Table 3. Occupational stress index aspects and total score among studied drivers.

\begin{tabular}{lcc}
\hline \multicolumn{1}{c}{$\begin{array}{c}\text { Occupational Stress Index (OSI) Aspects } \\
\text { (Maximum Score) }(\boldsymbol{n}=\mathbf{1 3 1})\end{array}$} & Mean \pm SD & Median(IQR) \\
\hline Total underload (20) & $9.56 \pm 2.58$ & $10.00(3.00)$ \\
Total high demand (30) & $16.92 \pm 2.58$ & $17.00(4.00)$ \\
Total strictness (21) & $17.22 \pm 1.61$ & $18.00(1.00)$ \\
Total extrinsic time pressure (9) & $7.56 \pm 1.38$ & $7.00(2.00)$ \\
Total noxious exposure (12) & $6.75 \pm 1.29$ & $7.00(2.00)$ \\
Total symbolic aversiveness (avoidance) (20) & $9.22 \pm 1.58$ & $9.00(2.00)$ \\
Total conflict (29) & $12.76 \pm 2.04$ & $12.00(3.00)$ \\
Total OSI score (141) & $79.98 \pm 6.14$ & $81.00(9.00)$ \\
\hline
\end{tabular}

IQR: Interquartile Range.

Table 4 demonstrates statistically significant differences among driver groups regarding underload, high demand, extrinsic time pressure, noxious exposure, strictness, and conflict aspects scores of OSI. Additionally, the total OSI score shows a statistically significant difference among car drivers, bus drivers, and truck drivers $(p=0.003)$. As Figure 1 shows, $79.4 \%$ of the drivers have dyslipidemia. 
Table 4. Occupational index stress among car, bus and truck drivers (Kruskal-Wallis test) $(n=131)$.

\begin{tabular}{|c|c|c|c|c|}
\hline OSI Aspects & $\begin{array}{c}\text { Car Drivers } \\
n=44\end{array}$ & $\begin{array}{c}\text { Bus Drivers } \\
n=43\end{array}$ & $\begin{array}{c}\text { Truck Drivers } \\
n=44\end{array}$ & $p$-Value \\
\hline Total underload & $8.50 \pm 2.11^{\wedge}$ & $9.12 \pm 2.12^{\#}$ & $11.07 \pm 2.76$ & $0.000 *$ \\
\hline Total high demand & $16.82 \pm 2.31^{\wedge}$ & $17.56 \pm 2.00^{\#}$ & $16.39 \pm 1.33$ & $0.020 *$ \\
\hline Total strictness & $16.36 \pm 2.27^{\wedge}$ & $17.58 \pm 1.07^{\#}$ & $17.58 \pm 0.62$ & $0.000 *$ \\
\hline $\begin{array}{c}\text { Total extrinsic time } \\
\text { pressure }\end{array}$ & $8.00 \pm 1.12 @$ & $7.44 \pm 1.42$ & $7.23 \pm 1.49$ & $0.046 *$ \\
\hline Total noxious exposure & $6.82 \pm 1.28^{\wedge} @$ & $5.72 \pm 0.96^{\#}$ & $7.68 \pm 0.74$ & $0.000 *$ \\
\hline Total symbolic aversiveness & $8.84 \pm 1.74$ & $9.44 \pm 1.80$ & $9.39 \pm 1.04$ & 0.189 \\
\hline Total conflict & $12.20 \pm 2.38^{\wedge}$ & $13.40 \pm 1.87$ & $12.68 \pm 1.67$ & $0.035 *$ \\
\hline Total OSI & $77.55 \pm 6.79^{\wedge} @$ & $80.26 \pm 6.02$ & $82.16 \pm 4.62$ & $0.003 *$ \\
\hline
\end{tabular}

OSI Occupational Stress Index, * Statistically significant at $p<0.05,{ }^{\wedge}$ Mann-Whitney test between car drivers is statistically significant at $p<0.05$ compared with bus drivers, ${ }^{\oplus}$ Mann-Whitney test between car drivers is statistically significant at $p<0.05$ compared with truck drivers, ${ }^{*}$ Mann-Whitney test between bus drivers is statistically significant at $p<0.05$ compared with truck drivers.

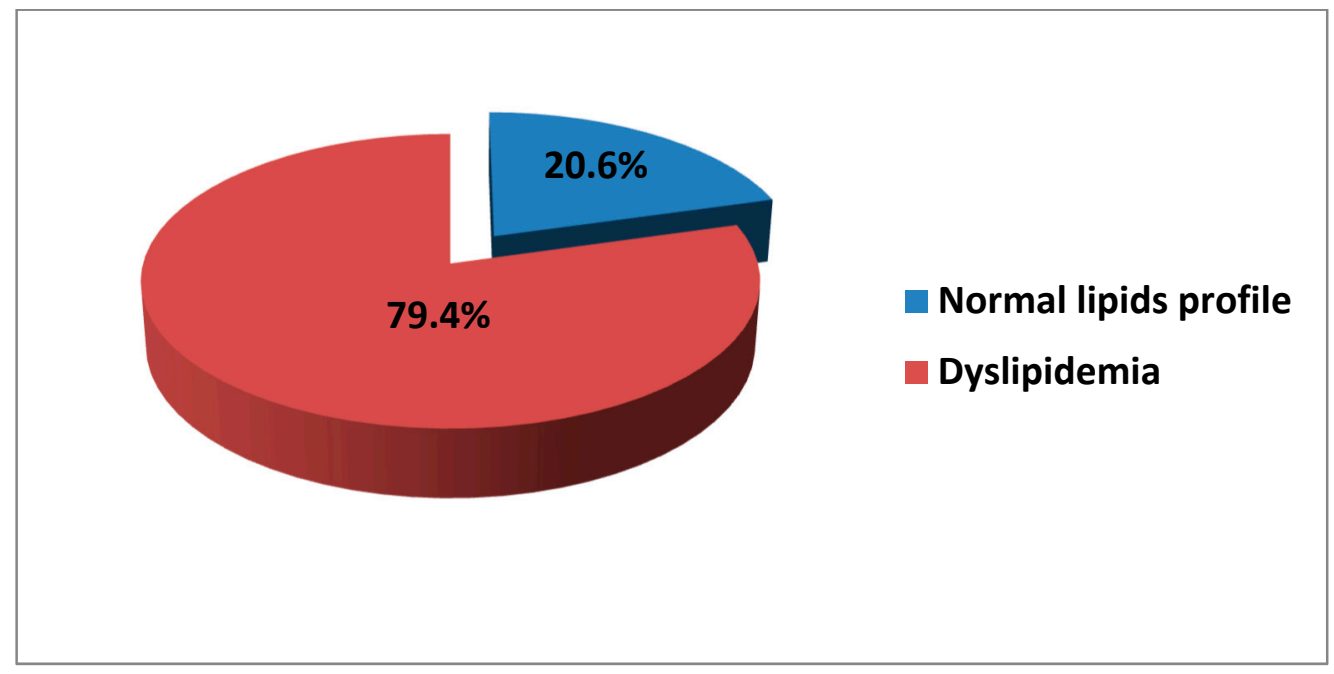

Figure 1. Prevalence of dyslipidemia among drivers $(n=131)$.

Table 5 shows that $51.9 \%$ of the studied drivers suffered from hypercholesterolemia. The mean of the cholesterol level was $204.37 \pm 37.01 \mathrm{mg} / \mathrm{dL}$. In addition, $37.4 \%$ had hypertriglyceridemia. The mean of the triglycerides was $146.79 \pm 90.86 \mathrm{mg} / \mathrm{dL}$. The LDL-C was high among $50.4 \%$ of the studied drivers. The mean of the LDL-C was $133.56 \pm 38.74 \mathrm{mg} / \mathrm{dL}$, and the HDL-C level was low among $45 \%$ of them. The mean of the HDL-C was $42.40 \pm 11.32 \mathrm{mg} / \mathrm{dL}$.

Table 5. Lipid profile of studied drivers $(n=131)$.

\begin{tabular}{|c|c|c|c|c|}
\hline \multirow{2}{*}{ Lipid profile (mg/dL) } & \multicolumn{2}{|c|}{ Normal } & \multicolumn{2}{|c|}{ Abnormal } \\
\hline & No. & $\%$ & No. & $\%$ \\
\hline Cholesterol & 63 & 48.1 & 68 & 51.9 \\
\hline Range & \multicolumn{4}{|c|}{$113-281$} \\
\hline Mean \pm SD & \multicolumn{4}{|c|}{$204.37 \pm 37.01$} \\
\hline Median (IQR) & \multicolumn{4}{|c|}{$205.00(49.00)$} \\
\hline Triglycerides & 82 & 62.6 & 49 & 37.4 \\
\hline Range & \multicolumn{4}{|c|}{$44-610$} \\
\hline Mean \pm SD & \multicolumn{4}{|c|}{$146.79 \pm 90.86$} \\
\hline Median (IQR) & \multicolumn{4}{|c|}{$126.00(94.00)$} \\
\hline LDL-C & 65 & 49.6 & 66 & 50.4 \\
\hline
\end{tabular}




\section{Range \\ Mean \pm SD \\ Median (IQR)}

HDL-C

Range

Mean \pm SD

Median (IQR)
54-229

$133.56 \pm 38.74$

133.00(56.00)

72

55.0

$$
\text { 22-88 }
$$

$42.40 \pm 11.32$

41.00(11.00)

LDL-C: Low-density lipoprotein cholesterol; HDL-C: High-density lipoprotein cholesterol; IQR: Interquartile Range.

To assess the lipid profile among the three driver groups, Figure 2 reveals that $70.5 \%$ of car drivers, $86 \%$ of bus drivers and $81.6 \%$ of truck drivers have dyslipidemia but that this difference is statistically insignificant.

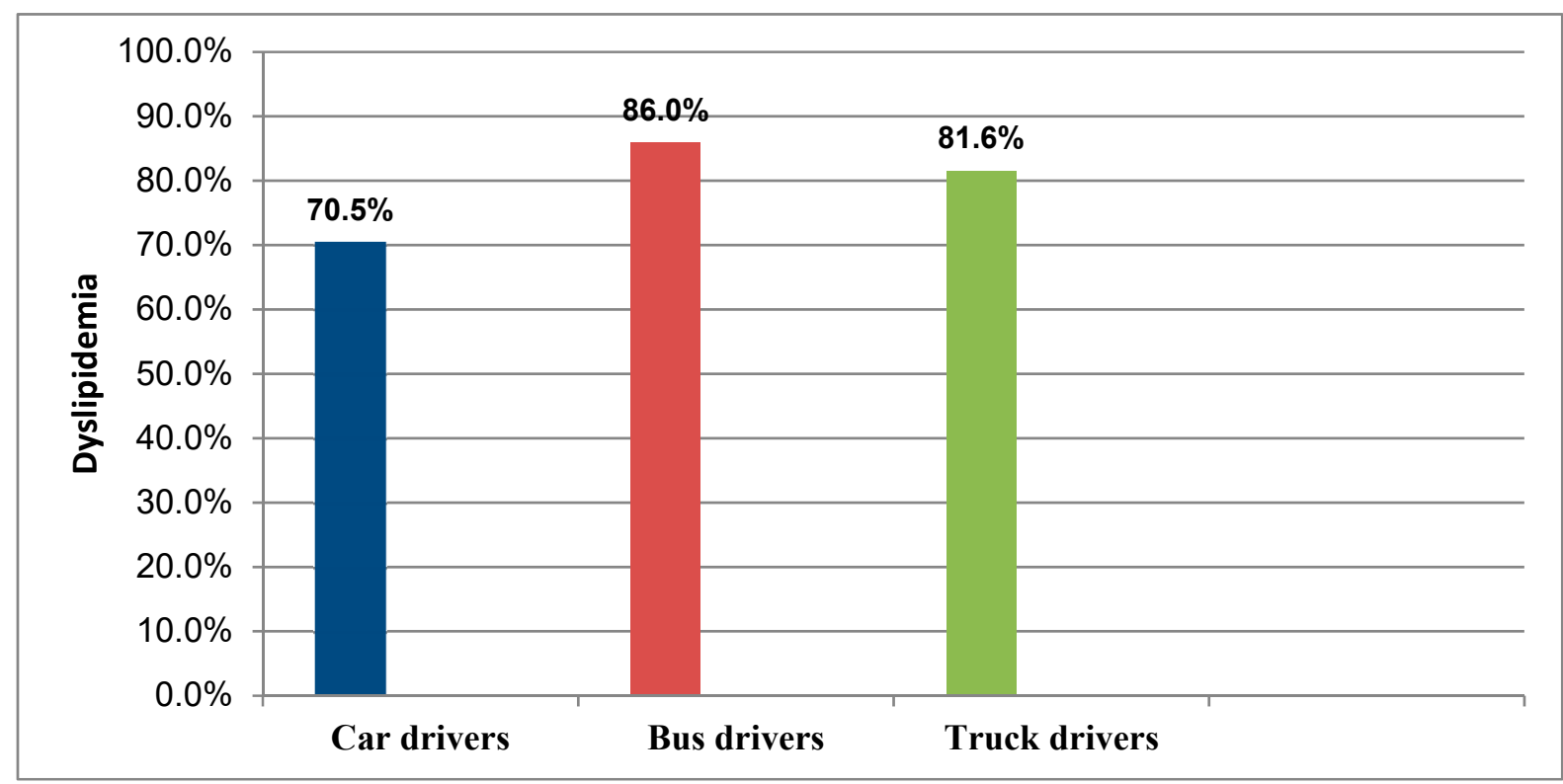

Figure 2. Comparison of prevalence of dyslipidemia among drivers' groups.

Regarding the cardiovascular risk factors scores, Table 6 shows that the family history of CVD score ranged from $0-45$. In addition, the lifestyle score ranged from 0-138. Besides, the range of sleep score was 0-22. Moreover, life-stress score ranged from $-10-89$ and blood-sugar score ranged from $0-110$. Furthermore, the diet-score range was 8-26.

Table 6. Cardiovascular risk factors scores among studied drivers $(n=131)$.

\begin{tabular}{lccc}
\hline Cardiovascular risk factors scores (Maximum score) & Range & Mean \pm SD & Median \\
\hline Family history of CVD (45) & $0-45$ & $12.25 \pm 12.45$ & 15.00 \\
Lifestyle (138) & $0-138$ & $68.95 \pm 39.57$ & 58.00 \\
Exercise practice (20) & $-25-20$ & $16.73 \pm 9.22$ & 20.00 \\
Smoking status (80) & $0-80$ & $33.51 \pm 36.12$ & 10.00 \\
Exposure to passive smoking (25) & $0-25$ & $14.50 \pm 12.39$ & 25.00 \\
Environment (13) & $0-9$ & $4.21 \pm 2.47$ & 3.00 \\
Sleep (24) & $0-22$ & $4.34 \pm 4.30$ & 3.00 \\
Life stress (331) & $-10-89$ & $5.12 \pm 14.68$ & 0.00 \\
Blood sugar (110) & $0-110$ & $11.60 \pm 29.06$ & 0.00 \\
Diet (48) & $-8-26$ & $6.33 \pm 7.63$ & 5.00 \\
\hline
\end{tabular}

CVD: cardiovascular disease. 
Regarding occupational risk factors of dyslipidemia, Table 7 reveals that average duration of driving hours per day is the only statistically significant risk factor associated with dyslipidemia with a mean of $6.00 \pm 2.69 \mathrm{hr}$ per day among drivers with normal lipids profiles compared with 7.20 $\pm 2.31 \mathrm{hr}$ per day among drivers with dyslipidemia. By contrast, OSI aspects, total OSI score, driving inside a city or between cities do not have a statistically significant relationship with dyslipidemia among the studied drivers.

Table 7. Occupational risk factors of dyslipidemia among drivers with normal lipid profile and drivers with dyslipidemia (Mann-Whitney U Test) $(n=131)$.

\begin{tabular}{lccc}
\hline \multicolumn{1}{c}{ Occupational Risk Factors } & No Dyslipidemia $(\boldsymbol{n}=\mathbf{2 7})$ & Dyslipidemia $(\boldsymbol{n}=\mathbf{1 0 4})$ & $\boldsymbol{p}$-Value \\
\hline Underload aspect & $9.00 \pm 2.45$ & $9.71 \pm 2.60$ & 0.170 \\
High-demand aspect & $17.11 \pm 1.91$ & $16.87 \pm 1.99$ & 0.508 \\
Strictness aspect & $17.41 \pm 2.02$ & $17.17 \pm 1.49$ & 0.931 \\
Extrinsic time pressure aspect & $7.41 \pm 1.42$ & $7.60 \pm 1.37$ & 0.500 \\
Noxious exposure aspect & $6.44 \pm 1.45$ & $6.83 \pm 1.24$ & 0.246 \\
Symbolic aversiveness aspect & $9.22 \pm 1.60$ & $9.22 \pm 1.58$ & 0.942 \\
Conflict aspect & $12.14 \pm 1.49$ & $12.91 \pm 2.14$ & 0.114 \\
Total OSI & $78.74 \pm 6.09$ & $80.31 \pm 6.14$ & 0.239 \\
Average driving hours a day & $6.0 \pm 2.7$ & $7.2 \pm 2.3$ & $\mathbf{0 . 0 1 7}$ \\
Years as professional driver & $12.3 \pm 8.3$ & $12.5 \pm 7.9$ & 0.308 \\
City & & & \\
$\quad$ Inside the city & $10(37.0 \%)$ & $26(25.0 \%)$ & \\
$\quad$ Between cities & $3(11.1 \%)$ & $30(28.8 \%)$ & 0.139 c \\
$\quad$ Both & $14(51.9 \%)$ & $48(46.2 \%)$ &
\end{tabular}

Table 8 shows that noxious exposure and conflict aspects of the OSI have weak positive significant correlations with cholesterol level $(r=0.163,0.162$, respectively). The correlations between OSI and triglycerides level are insignificant except a weak negative significant correlation between high demand aspect and triglyceride level $(r=-0.148)$. Weak positive significant correlations are shown between conflict aspect score and LDL-C $(r=0.234)$ and between noxious exposure aspect score and LDL-C level $(\mathrm{r}=0.149)$.

Table 8. Correlations between occupational stress index and lipid profile $(n=131)$.

\begin{tabular}{lcccc}
\hline \multirow{2}{*}{ Occupational Stress Index (OSI) Aspects } & \multicolumn{4}{c}{ Spearman Rank Correlation Coefficient (r) } \\
& Cholesterol & Triglycerides & LDL-C & HDL-C \\
\hline Underload & 0.086 & 0.033 & 0.079 & -0.048 \\
High demand & -0.072 & $-\mathbf{0 . 1 4 8} *$ & -0.034 & -0.002 \\
Strictness & 0.020 & 0.010 & -0.039 & 0.099 \\
Extrinsic time pressure & -0.061 & -0.019 & -0.020 & 0.024 \\
Noxious exposure & $\mathbf{0 . 1 6 3} \mathbf{3}^{*}$ & 0.112 & $\mathbf{0 . 1 4 9}$ & 0.057 \\
Symbolic aversiveness & -0.127 & -0.065 & -0.081 & 0.034 \\
Conflict & $\mathbf{0 . 1 6 2}$ * & -0.137 & $\mathbf{0 . 2 3 4}$ & 0.297 \\
Total OSI & $0.093^{\text {a }}$ & -0.086 & 0.104 & 0.044 \\
\hline
\end{tabular}

a Pearson correlation, ${ }^{*}$ Statistically significant at $p<0.05$.

With regards to non-occupational risk factors of dyslipidemia, Table 9 shows that $72.4 \%$ of drivers exposed to passive smoking had dyslipidemia compared with $27.6 \%$ who did not have dyslipidemia and that this difference is statistically significant. Moreover, the BMI mean is higher among dyslipidemic drivers $(29.79 \pm 4.06)$ compared with drivers having normal lipid profile $(27.73$ $\pm 4.44)$ and the difference is statistically significant. Other non-occupational factors do not have a statistically significant relationship with dyslipidemia. 
Table 9. Non-occupational risk factors of dyslipidemia $(n=131)$.

\begin{tabular}{lccc}
\hline \multicolumn{1}{c}{ Non-Occupational Risk Factors } & $\begin{array}{c}\text { No Dyslipidemia } \\
(\boldsymbol{n}=\mathbf{2 7})\end{array}$ & $\begin{array}{c}\text { Dyslipidemia } \\
(\boldsymbol{n}=\mathbf{1 0 4})\end{array}$ & $\boldsymbol{p}$-Value \\
\hline Age & $41.4 \pm 9.0$ & $39.3 \pm 8.1$ & $0.325^{\mathrm{a}}$ \\
Family history score & $12.78 \pm 12.27$ & $12.12 \pm 12.55$ & $0.753^{\mathrm{a}}$ \\
Lifestyle score & $66.00 \pm 39.04$ & $69.72 \pm 39.86$ & $0.709^{\mathrm{a}}$ \\
Exercise practice score & $15.81 \pm 11.05$ & $16.97 \pm 8.73$ & $0.638^{\mathrm{a}}$ \\
Smoking status & & & \\
Never & $14(51.9 \%)$ & $43(41.3 \%)$ & \\
Ex-smoker & $3(11.1 \%)$ & $12(11.5 \%)$ & \\
Current smoker fewer than 20 cigarettes/day & $4(14.8 \%)$ & $12(11.5 \%)$ & $0.592^{\mathrm{b}}$ \\
Current smoker equal or more than 20 & $6(22.2 \%)$ & $37(35.6 \%)$ & \\
cigarettes/day & $24.0 \pm 12.0$ & $27.5 \pm 10.7$ & $0.372^{\mathrm{a}}$ \\
Number of cigarettes/day & $15.9 \pm 7.1$ & $15.71 \pm 7.0$ & $0.896^{\mathrm{a}}$ \\
Years of smoking if current & $21(27.6 \%)$ & $55(72.4 \%)$ & $\mathbf{0 . 0 2 0 ^ { \mathrm { c } }}$ \\
Passive smoker & $4.00 \pm 2.45$ & $4.59 \pm 2.38$ & $0.637^{\mathrm{a}}$ \\
Environment score & $4.33 \pm 3.04$ & $4.86 \pm 4.29$ & $0.505^{\mathrm{a}}$ \\
Sleep score & $10.78 \pm 20.81$ & $5.47 \pm 16.31$ & $0.896^{\mathrm{a}}$ \\
Life-stress score & $6.67 \pm 21.30$ & $12.89 \pm 30.71$ & $0.430^{\mathrm{a}}$ \\
Blood-sugar score & $7.00 \pm 6.00$ & $9.00 \pm 6.91$ & $0.179^{\mathrm{d}}$ \\
Diet score & $27.73 \pm 4.44$ & $29.79 \pm 4.06$ & $\mathbf{0 . 0 2 3} \mathrm{d}^{*}$ \\
BMI & $121.1 \pm 14.5$ & $122.2 \pm 12.2$ & $0.839^{\mathrm{a}}$ \\
Systolic Blood Pressure & $78.5 \pm 9.9$ & $79.3 \pm 9.4$ & $0.995^{\mathrm{a}}$ \\
\hline Diastolic Blood Pressure & & & \\
\hline
\end{tabular}

BMI: Body mass index, a Mann-Whitney U test, ${ }^{\mathrm{b}}$ Fisher exact test, ${ }^{\mathrm{c}}$ Chi-squared test, ${ }^{\mathrm{d}}$ Student $\mathrm{t}$-test,

* Statistically significant at $p<0.05$.

Regarding independent significant risk factors of dyslipidemia, binary logistic regression analysis identified conflict aspect of the OSI, average duration of driving hours per day, and BMI as positive statistically significant independent risk factors for dyslipidemia (Table 10).

Table 10. Logistic regression of independent risk factors of dyslipidemia $(n=131)$.

\begin{tabular}{lcccc}
\hline \multicolumn{1}{c}{ Covariates of Dyslipidemia } & $\boldsymbol{B}$ & $\boldsymbol{p}$ Value & OR & $\mathbf{( 9 5 \% \text { CI } )}$ \\
\hline Strictness score & -0.296 & 0.087 & 0.744 & $(0.530-1.044)$ \\
Conflict score & 0.281 & $\mathbf{0 . 0 4 2}^{*}$ & 1.324 & $(1.010-1.736)$ \\
Average of driving hours a day & 0.346 & $\mathbf{0 . 0 0 2}^{*}$ & 1.414 & $(1.137-1.759)$ \\
BMI & 0.176 & $\mathbf{0 . 0 0 6 *}$ & 1.192 & $(1.052-1.351)$ \\
Constant & -4.428 & 0.199 & 0.012 & \\
& & $\mathbf{0 . 0 0 0}^{*}$ & & \\
\hline
\end{tabular}

BMI: Body mass index. Variables entered and excluded: underload aspect, high demand aspect, extrinsic time pressure aspect, noxious exposure aspect, symbolic averseness aspect, total occupational stress index score, smoking status, passive smoking status, and diet. * Statistically significant at $p<0.05$. Cox and Snell $\mathrm{R}$ Square $=0.153$. Nagelkerke $\mathrm{R}$ Square $=0.239$.

In the present study, the total OSI score mean was $79.98 \pm 6.14$ among the studied drivers. This agrees with the results of a Serbian study conducted on 417 male professional drivers, where total OSI was $63.9 \pm 8.8$ [1]. Professional drivers are exposed to occupational stress because driving is a stressful task. Occupational stress of drivers can also occur due to timetables and working hours, traffic and weather conditions, and passenger and goods safety [3].

The current study reveals that $79.4 \%$ of the studied drivers have dyslipidemia and $51.9 \%$ of them suffer from hypercholesterolemia, 37.4\% have hypertriglyceridemia. 50.4\% have high LDL-C and $45 \%$ have a low HDL-C level. This is in agreement with the results of an Iranian study 
conducted on 429 bus and truck male drivers where $53.4 \%$ had elevated triglyceride and $48.7 \%$ have low HDL-C levels [16]. Also, these findings partly support the results of a Korean study conducted on 443 male bus drivers, where $64.2 \%$ of them had high triglyceride, $38.6 \%$ had high cholesterol, $36.3 \%$ had low HDL-C, and $25.4 \%$ had high LDL-C [5].

By contrast, Biglari et al.'s (2016) results showed that $28.3 \%$ of the studied drivers had hypercholesterolemia with a cholesterol level of $>200 \mathrm{mg} / \mathrm{dL}$ and $15.2 \%$ had hypertriglyceridemia with a triglycerides level of $>240 \mathrm{mg} / \mathrm{dL}$ [17]. The difference in prevalence of dyslipidemia among studies may arise from the multifactorial etiology of dyslipidemia, which is a heterogeneous disorder that may occur due to multiple etiologies. Most of dyslipidemia is secondary to dietary habits and lifestyle. So, modern diet and lifestyle factors such as tobacco use, high fat intake, obesity, and sedentary activity play roles in the current epidemic of atherosclerosis [8].

Regarding the association of occupational stress and lipid profile, according to the results obtained in the present study, the total OSI score does not have a statistically significant relationship with dyslipidemia among studied drivers. Moreover, insignificant correlations are found between total OSI score and cholesterol level, triglycerides, LDL-C, or HDL-C. These results corroborate with findings from a meta-analysis conducted to investigate occupational stress and cardiovascular disease risk factors. No association was found between occupational stress and lipid profile [6]. The present study results also concur with Biglari et al. (2016) who did not reveal a significant relationship between job stress and dyslipidemia. In addition, the correlation tests do not demonstrate significant correlations between stress and blood cholesterol or triglycerides levels [18]. Moreover, Tsutsumi et al. (2003) concluded that job control or job strain (the ratio of demands to control) did not have a significant association with any of the lipid levels in males or females and suggest that psychologically demanding jobs may be related to an unfavorable lipid profile, but that the effect of job strain on atherogenic lipids is negligible [18].

This is in contrast to Jovanović et al.'s (2008) results, which reveal statistically significant relations between OSI and total cholesterol, LDL-C and triacylglycerol [1]. In addition, Djindjic et al.'s (2012) results found a significant association between total OSI score and any lipid profile abnormality. The group as a whole showed significant associations between total OSI and hypercholesterolemia, hypertriglyceridemia, and low (HDL) cholesterol [19]. Furthermore, Djindjić et al.'s (2013) results suggest a significant difference in prevalence of dyslipidemia associated with total OSI and some OSI aspects among professional drivers. Logistic regression analysis showed that total OSI score is significant risk factor for high triglycerides, high LDL-C, and low HDL-C [20].

\section{Conclusions}

The professional drivers in the study setting are exposed to occupational stress, with a high proportion having dyslipidemia. The total occupational stress index score does not have a statistically significant relationship with dyslipidemia, while certain aspects of occupational stress index have significant relations with lipid profile.

\section{References}

1. Jovanović, J.; Stefanović, V.; Stanković, D.N.; Bogdanović, D.; Kocić, B.; Jovanović, M.; Jovanović, J. Serum lipids and glucose disturbances at professional drivers exposed to occupational stressors. Cent. Eur. J. Public Health 2008, 16, 54-58.

2. Rosenthal, T.; Alter, A. Occupational stress and hypertension. J. Am. Soc. Hypertens. 2012, 6, 2-22.

3. Costa, G. Stress of driving: General overview. G. Ital. Med. Lav. Ergon. 2101, 34, 348-351.

4. Greenlund, K.J.; Kiefe, C.I.; Giles, W.H.; Liu, K. Associations of job strain and occupation with subclinical atherosclerosis: The CARDIA Study. Ann. Epidemiol. 2010, 20, 323-331.

5. Shin, S.Y.; Lee, C.G.; Song, H.S.; Kim, S.H.; Lee, H.S.; Jung, M.S.; Yoo, S.K. Cardiovascular disease risk of bus drivers in a city of Korea. Ann. Occup. Environ. Med. 2013, 25, 34.

6. Nagaya, T.; Yoshida, H.; Takahashi, H.; Kawai, M. Incidence of type-2 diabetes mellitus in a large population of Japanese male white-collar workers. Diabetes Res. Clin. Pract. 2006, 74, 169-174. 
7. Cicero, A.F.; Colletti, A. Combinations of phytomedicines with different lipid lowering activity for dyslipidemia management: the available clinical data. Phytomedicine 2016, 23, 1113-1118.

8. Gau, G.T.; Wright, R.S. Pathophysiology, diagnosis, and management of dyslipidemia. Curr. Probl. Cardiol. 2006, 31, 445-486.

9. Song, S.; Paik, H.Y.; Park, M.; Song, Y. Dyslipidemia patterns are differentially associated with dietary factors. Clin. Nutr. 2016, 35, 885-891. K.J.; Kiefe, C.I.; Giles, W.H.; Liu, K. Associations of job strain and occupation with subclinical atherosclerosis: The CARDIA Study. Ann. Epidemiol. 2010, 20, 323-331.

10. Belkic, K.; Savic, C. The occupational stress index--An approach derived from cognitive ergonomics applicable to clinical practice. Scand. J. Work Environ. Health 2008, 34, 169.

11. Sydney Natural Health and Life Style Clinic [Internet]. Forms, cardiovascular-risk-assessment-questionnaire.pdf. Available online: http://www.sydneynaturalhealth.com.au/uploads/1/6/1/3/16132402/ cardiovascular-risk-assessment-questionnaire.pdf (accessed on 10 May 2016).

12. Centers of disease control and prevention. High Blood Pressure 2014. Available online: http://www.cdc.gov/bloodpressure/measure.htm (accessed on 22 April 2016).

13. Centers of disease control and prevention. Division of Nutrition, Physical Activity, and Obesity 2015. Available online: http://www.cdc.gov/healthyweight/assessing/bmi/adult_bmi/ (accessed on 22 April 2016).

14. Grundy, S.M.; Cleeman, J.I.; Daniels, S.R.; Donato, K.A.; Eckel, R.H.; Franklin, BA.; Spertus, J.A. Diagnosis and management of the metabolic syndrome. Circulation 2005, 112, 2735-2752.

15. National Cholesterol Education Program. Expert Panel on Detection, Evaluation, and Treatment of High Blood Cholesterol in Adults (Adult Treatment Panel III): Third Report of the National Cholesterol Education Program (NCEP) Expert Panel on Detection, Evaluation, and Treatment of High Blood Cholesterol in Adults (Adult Treatment Panel III) final report. Circulation 2002, 106, 3143-3421.

16. Saberi, H.R.; Moravveji, A.R.; Fakharian, E.; Dehdashti, A.R. Prevalence of metabolic syndrome in bus and truck drivers in Kashan, Iran. Diabetol. Metab. Syndr. 2011, 3, 8.

17. Biglari, H.; Ebrahimi, M.H.; Salehi, M.; Poursadeghiyan, M.; Ahmadnezhad, I.; Abbasi, M. Relationship between occupational stress and cardiovascular diseases risk factors in drivers. Int. J. Occup. Med. Environ. Health 2016, 29, 895-901.

18. Tsutsumi, A.; Kayaba, K.; Ishikawa, S.; Gotoh, T.; Nago, N.; Yamada, S.; Hayasaka, S. Job characteristics and serum lipid profile in Japanese rural workers: the Jichi Medical School Cohort Study. J. Epidemiol. 2003, 13, 63-71.

19. Djindjic, N.; Jovanovic, J.; Djindjic, B.; Jovanovic, M.; Jovanovic, J.J. Associations between the occupational stress index and hypertension, type 2 diabetes mellitus, and lipid disorders in middle-aged men and women. Ann. Occup. Hyg. 2012, 56, 1051-1062.

20. Djindjić, N.; Jovanović, J.; Đinđić, B.; Jovanović, M.; Pešić, M.; Jovanović, J.J. Work stress related lipid disorders and arterial hypertension in professional drivers: A cross-sectional study. Vojnosanitetski pregled 2013, 70, 561-568.

(C) 2018 by the authors. Licensee MDPI, Basel, Switzerland. This article is an open access article distributed under the terms and conditions of the Creative Commons Attribution (CC BY) license (http://creativecommons.org/licenses/by/4.0/). 